\title{
A Doherty Power Amplifier with Large Back-Off Power Range Using Integrated Enhancing Reactance
}

\author{
Wa Kong, ${ }^{1}$ Jing Xia $\left(\mathbb{D},{ }^{1,2}\right.$ Fan Meng, ${ }^{2}$ Chao Yu $\left(\mathbb{D},{ }^{2}\right.$ Lixia Yang, ${ }^{1}$ and Xiaowei Zhu ${ }^{2}$ \\ ${ }^{1}$ School of Computer Science and Communication Engineering, Jiangsu University, Zhenjiang 212013, China \\ ${ }^{2}$ State Key Laboratory of Millimeter Waves, Southeast University, Nanjing 210096, China \\ Correspondence should be addressed to Jing Xia; ujsasan@gmail.com
}

Received 21 December 2017; Accepted 11 March 2018; Published 15 April 2018

Academic Editor: Shichang Chen

Copyright (c) 2018 Wa Kong et al. This is an open access article distributed under the Creative Commons Attribution License, which permits unrestricted use, distribution, and reproduction in any medium, provided the original work is properly cited.

\begin{abstract}
A symmetric Doherty power amplifier (DPA) based on integrated enhancing reactance (IER) was proposed for large back-off applications. The IER was generated using the peaking amplifier with the help of a desired impedance transformation in the lowpower region to enhance the back-off efficiency of the carrier amplifier. To convert the impedances properly, both in the lowpower region and at saturation, a two-impedance matching method was employed to design the output matching networks. For verification, a symmetric DPA with large back-off power range over $2.2-2.5 \mathrm{GHz}$ was designed and fabricated. Measurement results show that the designed DPA has the $9 \mathrm{~dB}$ back-off efficiency of higher than $45 \%$, while the saturated output power is higher than $44 \mathrm{dBm}$ over the whole operation bandwidth. When driven by a $20 \mathrm{MHz}$ LTE signal, the DPA can achieve good average efficiency of around $50 \%$ with adjacent channel leakage ratio of about $-50 \mathrm{dBc}$ after linearization over the frequency band of interest. The linearity improvement of the DPA for multistandard wireless communication system was also verified with a dual-band modulated signal.
\end{abstract}

\section{Introduction}

In future wireless communication systems, such as the 5th generation of mobile communication network (5G), the ever increasing demand for high transmission data rate results in the employment of wideband and multiband modulated signals characterized by high peak-to-average power ratios (PAPRs). To efficiently amplify these signals at back-off power (BOP) range, the Doherty power amplifier (DPA) has garnered great research attention because of its significant efficiency enhancement and ease of configuration [1-6]. Conventional symmetric DPAs, employing the same transistors to design the carrier and peaking amplifiers, always achieve high-efficiency operation over $6 \mathrm{~dB}$ or less BOP range [712], which can hardly satisfy the efficiency requirement when wideband or multiband modulated signals with the PAPRs of higher than $6 \mathrm{~dB}$ are employed.

To further improve the back-off efficiency, various DPAs, such as asymmetric DPAs and multistage DPAs, have been investigated to achieve large BOP range [13-16]. These two kinds of DPAs utilize excessive modulation current of the peaking amplifier to achieve enlarged load span of the carrier amplifier for extended BOP range, leading to tradeoffs between design complexity, cost, and power utilization factor. To overcome the disadvantages of the aforementioned DPAs, modified symmetric DPAs have been proposed recently to attain larger than $6 \mathrm{~dB}$ high-efficiency range, which employ complex combining load technique [17] or maintain full voltage and current swings of both carrier and peaking amplifiers [18]. However, these approaches might suffer from complicated design methodology. Recently, the effect of the output impedance of the peaking stage has been investigated to extend the high-efficiency power range or bandwidth [1921] in DPA designs. In [21], we have proposed a novel method to extend the bandwidth of the DPA based on integrated compensating reactance, which was chosen near the opencircuit area in Smith chart. However, after further analysis, it is found that a reactance near the short-circuit point can also be used to enhance the back-off efficiency and thus extend the BOP range. 


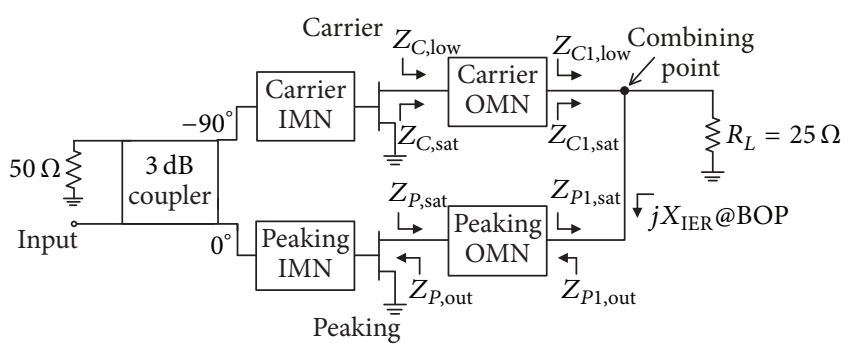

FIGURE 1: Simplified schematic of the proposed DPA.

In this paper, after analyzing the effect of a reactive load at the combining point on the back-off efficiency of the carrier amplifier, a new symmetric DPA with large BOP range based on enhancing reactance integrated in the peaking amplifier is proposed. This integrated enhancing reactance (IER) was obtained by using a two-impedance matching method to extend the BOP range. Unlike conventional DPA, the output impedance of the peaking amplifier in the proposed design has a lower reactance value for efficiency enhancement when the peaking amplifier is in off-state. For verification, a 2.2-2.5 GHz symmetric DPA with higher than $45 \%$ efficiency over $9 \mathrm{~dB}$ BOP range was designed. Experimental results of the output power and efficiency as well as the linearization results are also presented.

\section{Proposed DPA for BOP Range Extension}

The simplified schematic of the proposed DPA is shown in Figure 1, which comprises the carrier and peaking amplifiers, an input power divider, and a common load $R_{L}$. In Figure $1, Z_{C \text {,sat }}, Z_{C 1 \text {,sat }}, Z_{P \text {,sat }}$, and $Z_{P 1 \text {,sat }}$ represent the carrier and peaking load impedances at the device output and at the combining point when the DPA is at saturation, while $Z_{P \text {,out }}, Z_{P 1 \text {,out }}, Z_{C \text {,low }}$, and $Z_{C 1 \text {,low }}$ are the output and load impedances before the peaking amplifier turns on, respectively. Moreover, $X_{\text {IER }}$ denotes the IER generated by the peaking amplifier at BOP range for back-off efficiency enhancement. At saturation, the IER would not introduce undesirable effect on load modulation of the DPA when both carrier and peaking amplifiers turn on. The design procedure of the proposed DPA is presented as follows.

\subsection{Effect of Shunted Reactance on Carrier Back-Off Efficiency.}

To analyze the effect of the IER on the back-off efficiency of the carrier amplifier, the carrier output matching network (OMN) should be designed firstly. During the Doherty operation, the carrier OMNs should convert the load impedances to the desired values to obtain proper Doherty behavior.

By using load pull simulations based on large signal model of Wolfspeed CGH40010F GaN HEMT, the load impedances of $18+j 2 \Omega$ and $22+j 15 \Omega$ were chosen as $Z_{C \text {,sat }}$ and $Z_{C \text {,low }}$ at the frequency of $2.5 \mathrm{GHz}$, respectively. With the impedances $Z_{C 1 \text {, sat }}=50 \Omega$ and $Z_{C 1 \text { low }}=25 \Omega$, the carrier OMN can be designed by using stepped-impedance matching network theory [21], as shown in Figure 2. To evaluate performance over wide operation band, the simulated

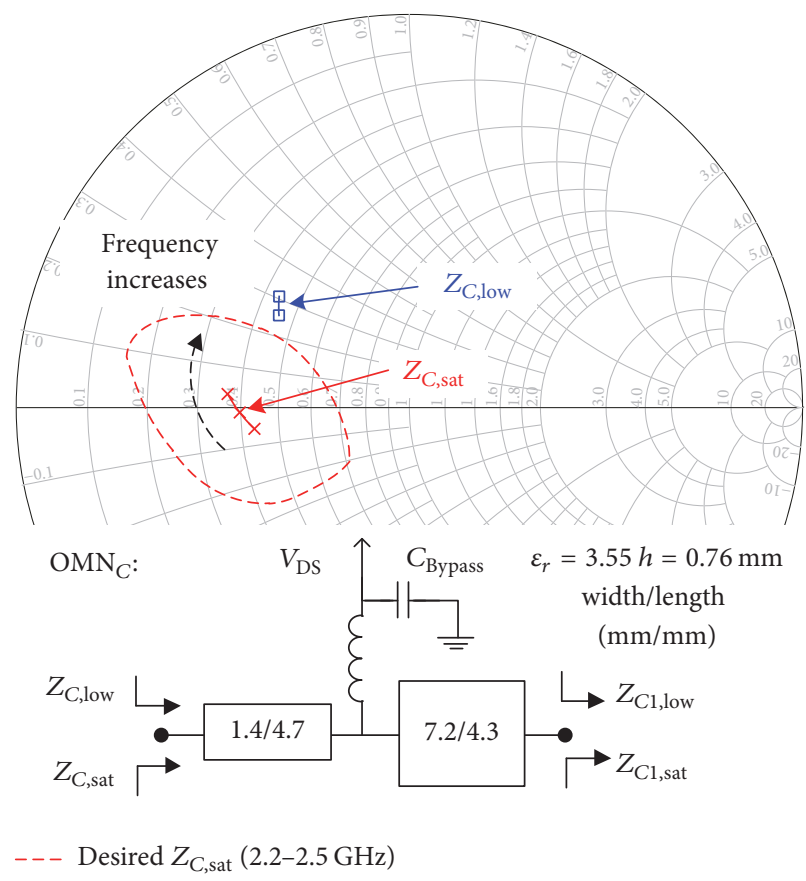

FIgURE 2: Designed carrier OMNs and the simulated impedances over the frequency band of $2.2-2.5 \mathrm{GHz}$.

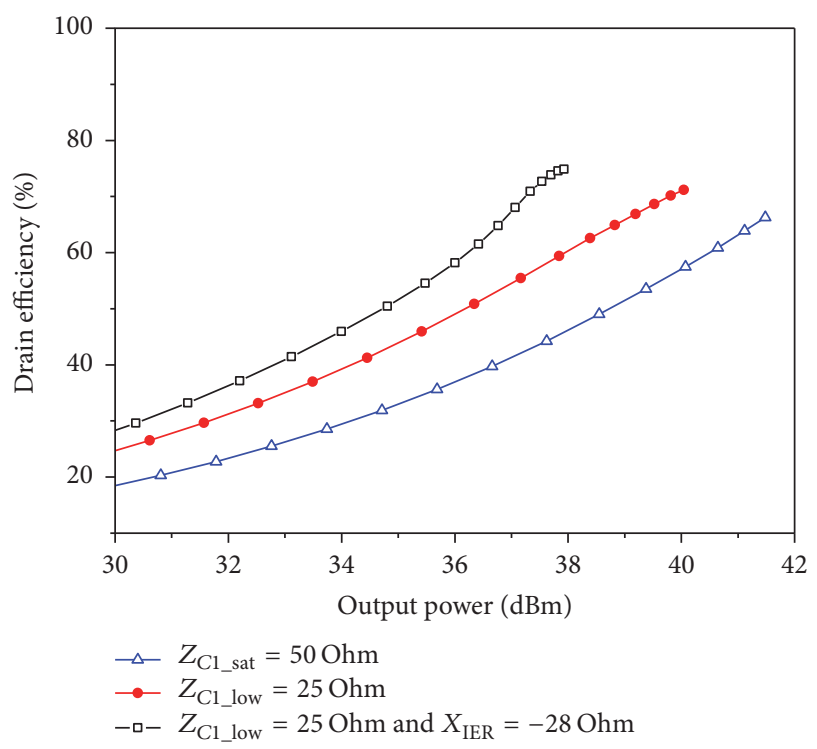

FIGURE 3: Simulated efficiency of the carrier amplifier versus output powers under different loads at $2.5 \mathrm{GHz}$.

impedances between 2.2 and $2.5 \mathrm{GHz}$ are also given. After the OMN was designed, the input matching network (IMN) can also be designed to cover the required bandwidth.

Figure 3 illustrates simulated drain efficiency of the carrier amplifier versus output powers with $Z_{\mathrm{Cl} \text {,sat }}=50 \Omega$ and $Z_{C 1 \text {,low }}=25 \Omega$ at $2.5 \mathrm{GHz}$. It is shown that, with the load impedance of $25 \Omega$, the carrier amplifier can achieve enhanced efficiency at back-off powers, which implies a BOP 


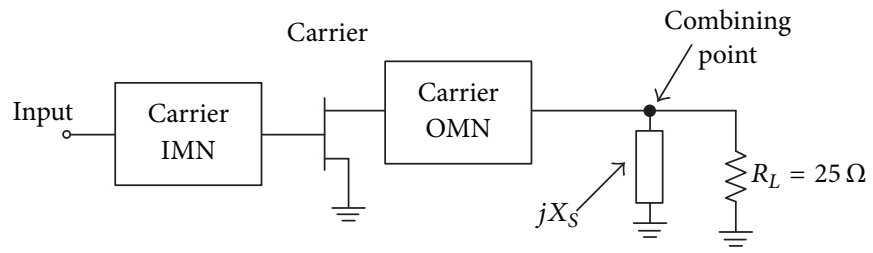

FIGURE 4: Carrier amplifier with a shunted reactance at the combining point.

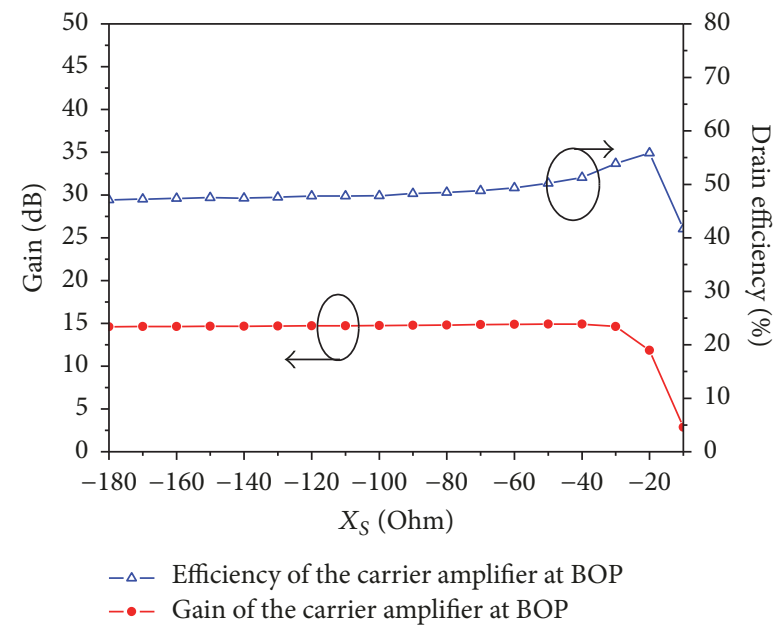

FIGURE 5: Back-off efficiency and gain of the carrier amplifier at $35 \mathrm{dBm}$ output power versus $X_{S}$.

range of about $6 \mathrm{~dB}$ in conventional symmetric DPA theoretically. However, to achieve larger than $6 \mathrm{~dB} B O P$ range, the carrier back-off efficiency should be further enhanced. In the proposed DPA, a shunted reactance at the combining point was employed to achieve high efficiency of the carrier amplifier in the low-power region. To determine the value of this shunted reactance $\left(X_{S}\right)$, using the schematic in Figure 4, the effect of the reactance on the carrier back-off efficiency was analyzed. Figure 5 depicts the back-off efficiency of the carrier amplifier and the gain performance at $35 \mathrm{dBm}$ output power versus the shunted reactance $X_{S}$ at $2.5 \mathrm{GHz}$. It can be observed that, with $X_{S}$ ranging from -36 to $-20 \Omega$, the efficiency of the carrier amplifier remains above 52\%, which corresponds to $9 \mathrm{~dB}$ BOP range in the proposed DPA. However, when the reactance increases to $-20 \Omega$, there is a large degradation of the gain performance.

After a trade-off between the back-off efficiency and gain performance, the desired value of the shunted reactance was chosen to be $-28 \Omega$. To illustrate the effect of the shunted reactance, simulated carrier efficiency with $Z_{C 1 \text {, low }}=25$ and $X_{S}=$ $-28 \Omega$ was also depicted in Figure 3 . When compared with the case without the shunted reactance, significant efficiency enhancement can be observed in the low-power region, which means larger BOP range can be achieved. However, according to Doherty operation principle, if an additional shunted reactance at the combining point is employed as the enhancing reactance for efficiency improvement, the

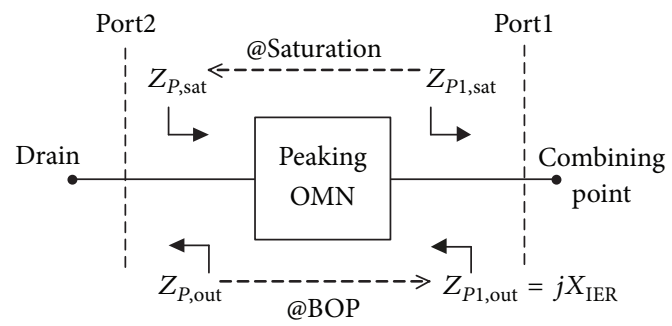

FIGURE 6: Desired impedance transformation of the peaking OMN.

load modulation at saturation will be improper, leading to unavoidable degradation of the output power and efficiency.

To solve this problem, in this paper, the output impedance of the peaking amplifier in off-state was employed to generate this enhancing reactance, which is integrated in the peaking amplifier output, that is, the IER. It should be mentioned that, unlike conventional DPA, the peaking output impedance in the proposed DPA is not an open circuit but a reactance in the low-power region. To obtain desired IER, a two-impedance matching method was employed to design the OMN of the peaking amplifier, as introduced in the following section.

2.2. Generation of IER for BOP Range Extension. As mentioned above, the IER used to extend the BOP range of the DPA was generated by the peaking amplifier before it turns on. The OMN of the peaking amplifier should satisfy the desired impedance transformation, as illustrated in Figure 6. The peaking OMN should convert the output impedance $Z_{P, \text { out }}$ to desired value of $Z_{P 1 \text {,out }}$ to generate the IER at BOP, while transforming $Z_{P 1 \text {,sat }}$ to $Z_{P \text {,sat }}$ at saturation, which can be achieved using the following design method.

According to network analysis, if the $S$ parameters of a lossless reciprocal OMN can be expressed in terms of $S_{22}$ and the phase of $S_{21}\left(\theta_{21}\right)$, the $A B C D$ parameters can also be determined by these two parameters [21]:

$$
\left[\begin{array}{ll}
A & B \\
C & D
\end{array}\right]=\left[\begin{array}{cc}
\frac{a+c}{2 \sqrt{c}} & Z_{0} \frac{b-c}{2 \sqrt{c}} \\
\frac{1}{Z_{0}} \frac{d-c}{2 \sqrt{c}} & \frac{e+c}{2 \sqrt{c}}
\end{array}\right],
$$

where $Z_{0}$ is the reference impedance and

$$
\begin{aligned}
& a=\left(1-S_{22}^{*} e^{j 2 \theta_{21}}\right)\left(1-S_{22}\right) \\
& b=\left(1-S^{*}{ }_{22} e^{j 2 \theta_{21}}\right)\left(1+S_{22}\right) \\
& c=\left(1-\left|S_{22}\right|^{2}\right) e^{j 2 \theta_{21}}
\end{aligned}
$$




$$
\begin{aligned}
d & =\left(1+S^{*}{ }_{22} e^{j 2 \theta_{21}}\right)\left(1-S_{22}\right) \\
e & =\left(1+S^{*}{ }_{22} e^{j 2 \theta_{21}}\right)\left(1+S_{22}\right) .
\end{aligned}
$$

Considering the peaking $\mathrm{OMN}$ in Figure 6, the impedances at $\mathrm{BOP}$ region and at saturation can be expressed as

$$
\begin{gathered}
Z_{P 1 \text {,out }}=\frac{Z_{P, \text { out }} A+B}{Z_{P, \text { out }} C+D} \\
Z_{P 1 \text {,sat }}^{*}=\frac{Z_{P, \text { sat }}^{*} A+B}{Z_{P, \text { sat }}^{*} C+D} .
\end{gathered}
$$

By means of (1) and (3), the following expressions are obtained:

$$
\begin{aligned}
& Z_{P 1 \text {,out }}=\frac{Z_{0} Z_{P, \text { out }} a+Z_{0}^{2} b+Z_{0}\left(Z_{P, \text { out }}-Z_{0}\right) c}{Z_{P, \text { out }} d+Z_{0} e+\left(Z_{0}-Z_{P, \text { out }}\right) c} \\
& \frac{Z_{0} Z_{P, \text { sat }}^{*} a+Z_{0}^{2} b+Z_{0}\left(Z_{P, \text { sat }}^{*}-Z_{0}\right) c}{Z_{P 1 \text {,sat }}^{*} Z_{P, \text { sat }}^{*} d+Z_{P 1 \text { sat }}^{*} Z_{0} e+Z_{P 1 \text { sat }}^{*}\left(Z_{0}-Z_{P, \text { sat }}^{*}\right) c} \\
& =1 .
\end{aligned}
$$

According to (4) and (5), if the impedances $Z_{P 1 \text {,out }}, Z_{P \text {,out }}$, $Z_{P 1 \text {,sat }}$, and $Z_{P \text {, sat }}$ are obtained, the design parameters $S_{22}$ and $\theta_{21}$ can be determined through solving these equations theoretically. Then, the OMN can be designed accordingly.

In the peaking OMN design, the optimum load impedance $Z_{P \text {, sat }}$ at $2.5 \mathrm{GHz}$ was obtained to be $19+j 5 \Omega$ by using ADS load pull simulations with large signal model of CGH40010F GaN HEMT under class-C bias condition. To determine the output impedance $Z_{P \text {,out }}$ when the peaking amplifier is in off-state, the transistor model was simulated without output matching network under small input signal stimulation in ADS large signal $S$-parameter simulation. According to the result, the output impedance $Z_{P \text {,out }}$ was found to be $0.5-j 36 \Omega$. Considering the phase of $\theta_{21}$ as a degree of freedom, using $Z_{P 1 \text {, sat }}=50 \Omega$, the parameter $S_{22}$ can then be calculated using (5), and a set of output impedance $Z_{P 1 \text {,out }}$ can be plotted, as shown in Figure 7. According to Section 2.1, the desired value of IER $\left(X_{\text {IER }}\right)$ is $-28 \Omega$. From Figure 7 , when $Z_{P 1 \text {,out }}$ is close to $-j 28 \Omega$, a series of $\theta_{21}=-36^{\circ}-n * 180^{\circ}(n=0,1,2, \ldots)$ can be obtained according to the periodicity of exponential function. Considering practical peaking OMN design, the $\theta_{21}$ was chosen to be $-216^{\circ}$, while the corresponding $S_{22}$ is calculated to be $-0.45+j 0.1$.

Considering the obtained $S$ parameters, the peaking OMN can then be designed by using ADS optimization, as shown in Figure 8. The load impedances at saturation power and the peaking output impedances in class- $\mathrm{C}$ operation are also depicted. The results show that, using the twopoint matching technique, the designed OMN can satisfy the impedance transformation of the peaking amplifier. Meanwhile, desired $X_{\text {IER }}$ of $-28 \Omega$ can be generated by using the peaking OMN.

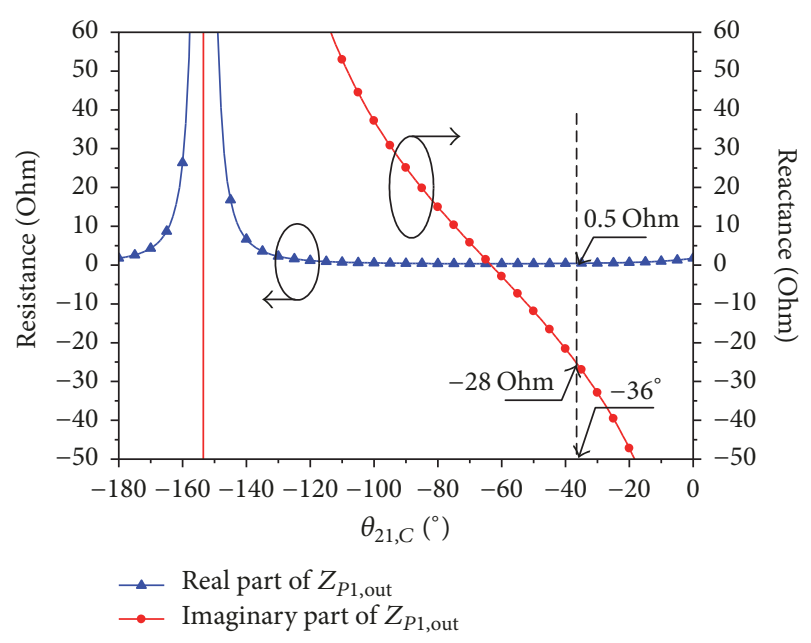

FIGURE 7: Graphical illustration of the two-impedance matching technique for the peaking OMN.

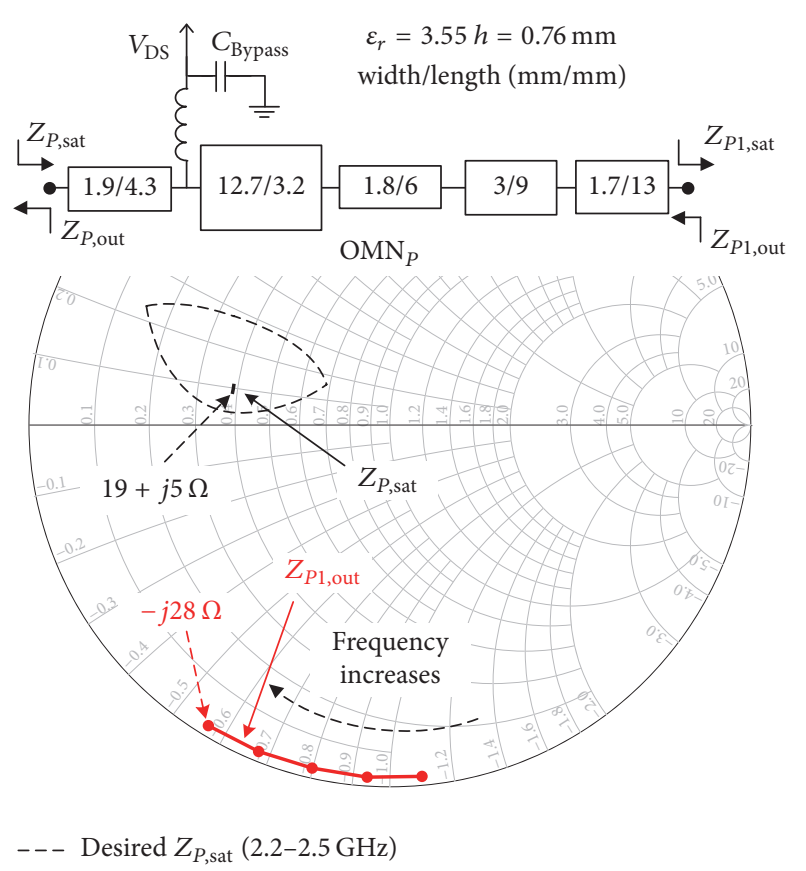

FIGURE 8: Designed peaking OMN and simulated impedances over the frequency band of $2.2-2.5 \mathrm{GHz}$.

2.3. DPA Design and Simulations. To verify the proposed method, a $2.2-2.5 \mathrm{GHz}$ symmetric DPA with IER for BOP range extension was designed using a Taconic RF35 substrate with $\varepsilon_{r}=3.55$ and thickness of $0.76 \mathrm{~mm}$. Figure 9 depicts the output network topology of the DPA, including the designed OMNs and a postmatching network (PMN) for impedance transformation between the output load of $50 \Omega$ and the common load of $25 \Omega$. Both the carrier and peaking IMNs are designed by using stepped-impedance matching network theory to cover the required frequency band, as shown in Figure 10. 


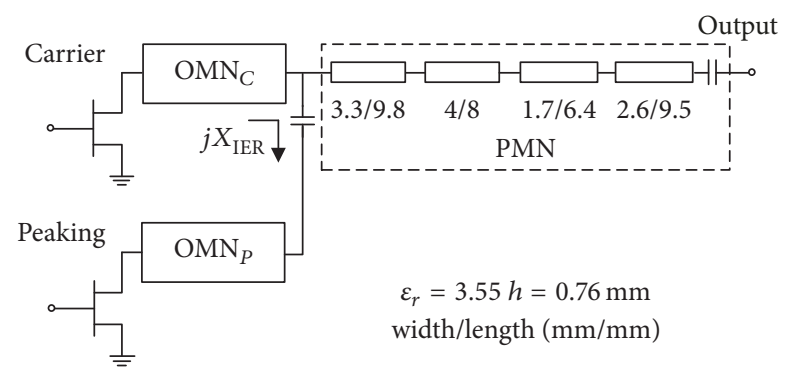

FIGURE 9: Topology of the output network of the DPA.

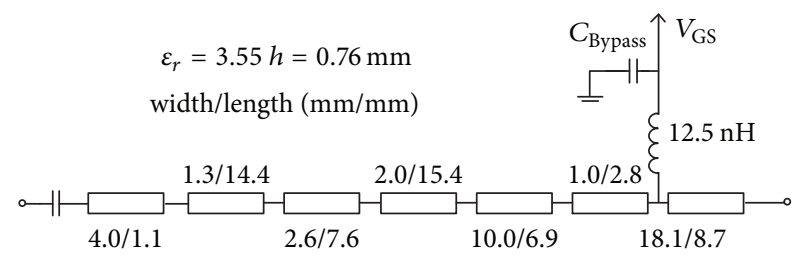

FIGURE 10: Designed IMN for the carrier and peaking amplifiers.

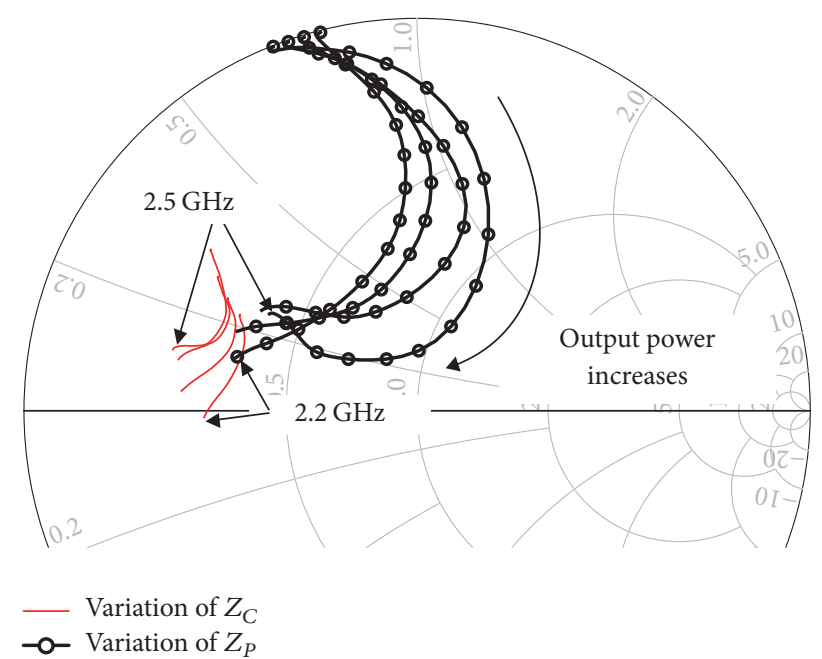

FIGURE 11: Simulated load traces of the carrier and peaking device in the Doherty operation.

After the output network and the IMNs were designed, using a $3 \mathrm{~dB} 90^{\circ}$ hybrid coupler as the input power splitter, the whole DPA can be designed and simulated. For comparative purposes, conventional DPA using similar IMNs and OMNs with the peaking output impedance of quasi open circuit was also simulated. In both the proposed and conventional DPAs, the carrier amplifier was in a class-AB bias condition with $I_{\mathrm{DS}}=0.05 \mathrm{~A}$, while the peaking one was biased in class- $\mathrm{C}$ mode with $V_{\mathrm{GS}}=-5.5 \mathrm{~V}$. For improved load modulation, the drain voltages of the carrier and peaking amplifiers were chosen to be 26 and $30 \mathrm{~V}$ so that these two amplifiers can generate similar output powers at saturation. In Figure 11, the simulated load impedance traces of the carrier and peaking device are depicted. The load impedances of both carrier and

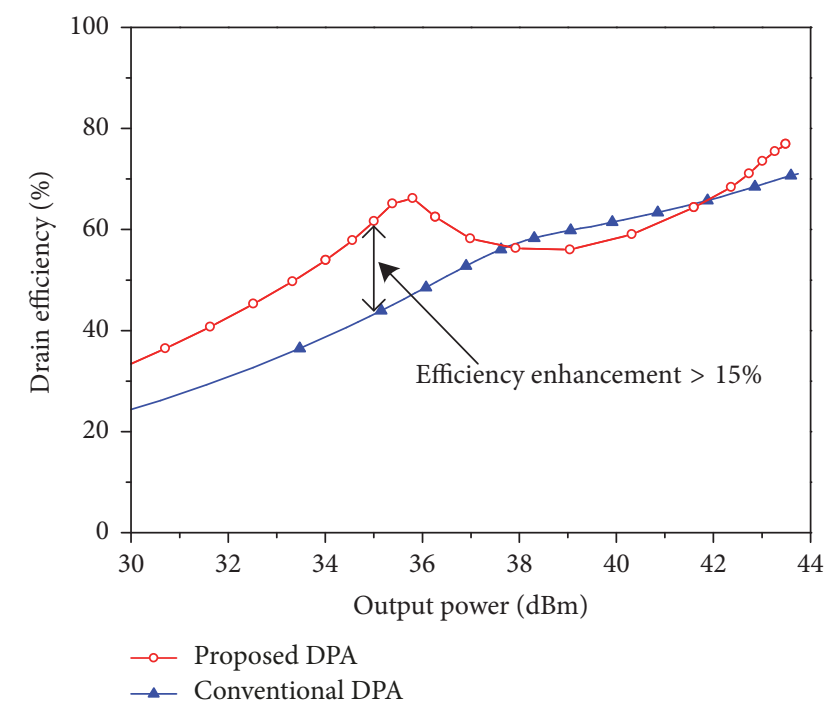

Figure 12: Simulated drain efficiencies of the proposed and conventional DPAs as a function of output power at $2.5 \mathrm{GHz}$.

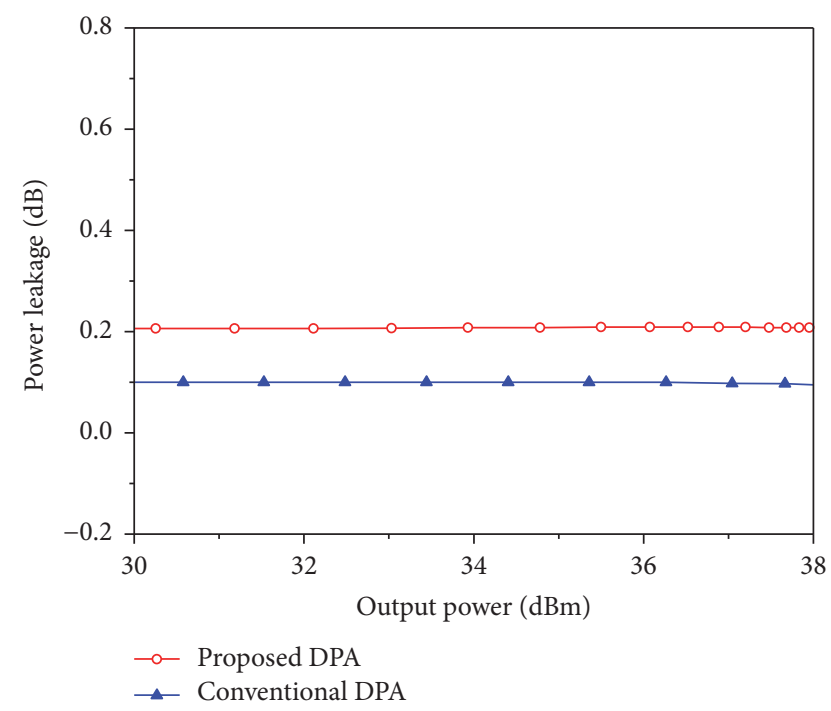

FIGURE 13: Simulated power leakages of the carrier amplifier in proposed and conventional DPAs.

peaking devices at saturation thus still satisfy the desired load impedance shown in Figures 2 and 8.

In Figure 12, the simulated drain efficiencies of the proposed and conventional DPAs are depicted. For the proposed $\mathrm{DPA}$, the simulated efficiency is $58 \%$ at $9 \mathrm{~dB}$ BOP which is $15 \%$ higher than the one of conventional DPA. To analyze the influence of this IER on the overall performance of the DPA, the power leakages of the carrier amplifier at the combining point were simulated, as shown in Figure 13. For the proposed DPA using IER, less than $0.1 \mathrm{~dB}$ power leakage increment can be observed when compared with conventional DPA. The efficiency enhancement confirms that expected BOP range extension can be achieved using the proposed IER, while the output power performance is similar to conventional design. 


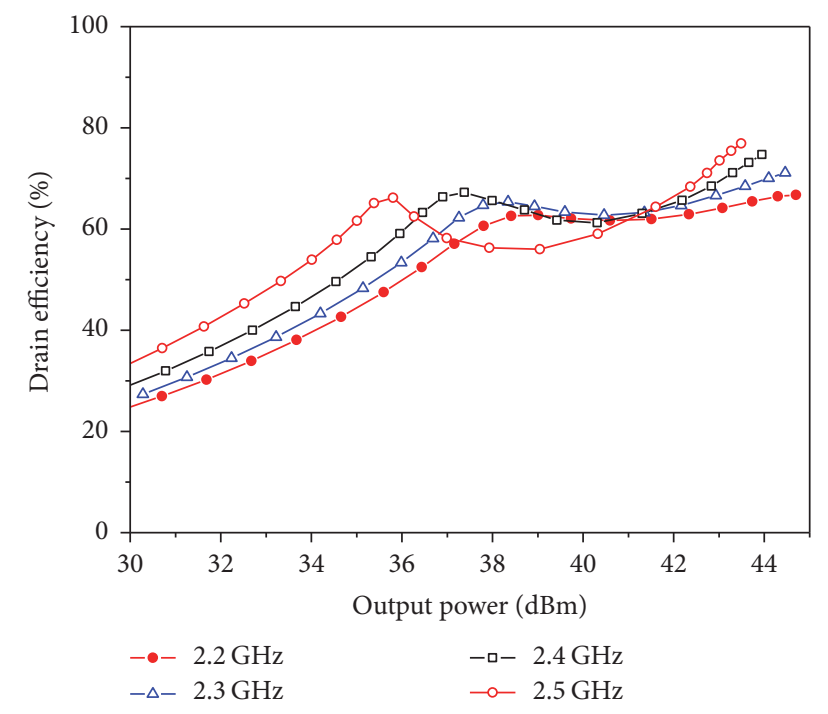

FIGURE 14: Simulated drain efficiencies of the proposed DPA at the frequencies of 2.2, 2.3, 2.4, and $2.5 \mathrm{GHz}$.

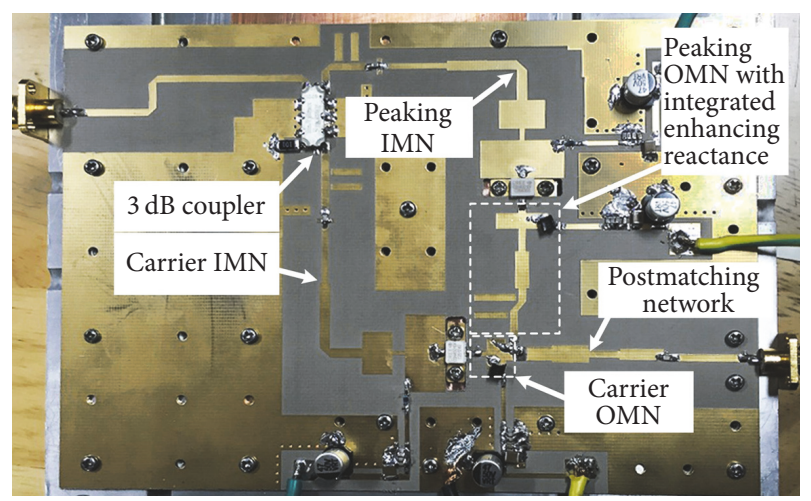

FIgURE 15: Photograph of the fabricated DPA.

To evaluate the efficiency over wide frequency band, the simulated drain efficiencies of the proposed DPA at 2.2, 2.3, 2.4, and $2.5 \mathrm{GHz}$ are also given in Figure 14. The drain efficiencies are $48 \%-58 \%$ and $66 \%-75 \%$ for the $9 \mathrm{~dB}$ BOP and saturation regions, respectively. In addition, the efficiency plateau in large BOP range, which verifies the high-efficiency range extension based on IER, can also be observed.

\section{Realization and Experimental Results}

For experimental validation, the proposed DPA with large BOP range based on IER was fabricated, as shown in Figure 15. The measurement results using continuous wave signals and modulated signals are given as follows.

Figure 16 shows measured drain efficiencies and gains versus output powers at $2.2,2.3,2.4$, and $2.5 \mathrm{GHz}$ under continuous wave measurements. The designed DPA maintains drain efficiency of $45 \%-52 \%$ at about $9 \mathrm{~dB}$ BOP over the frequency band of interest. The peak output power ranges from 44 to $44.6 \mathrm{dBm}$ with maximum efficiency of $63 \%-68 \%$. One can observe that the drain efficiency can maintain

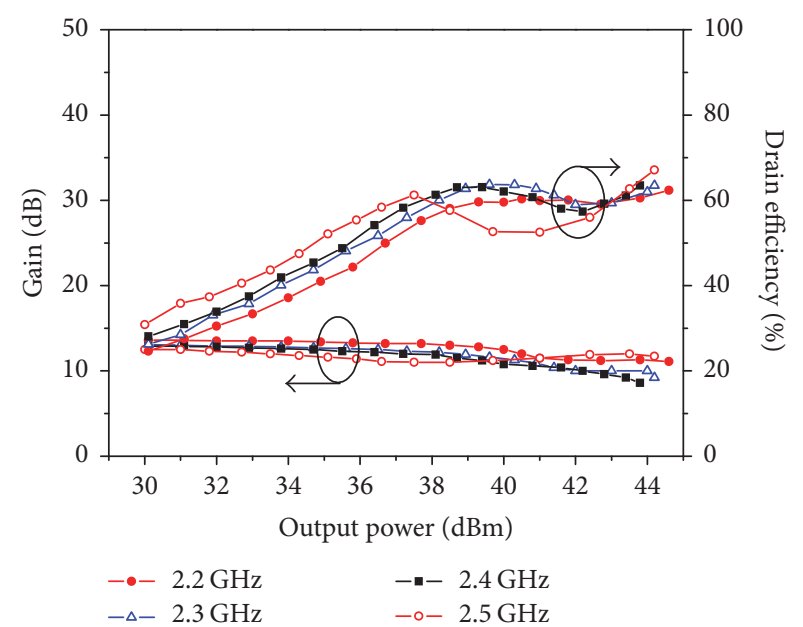

FIGURE 16: Measured drain efficiency and gain versus output power at different frequencies.

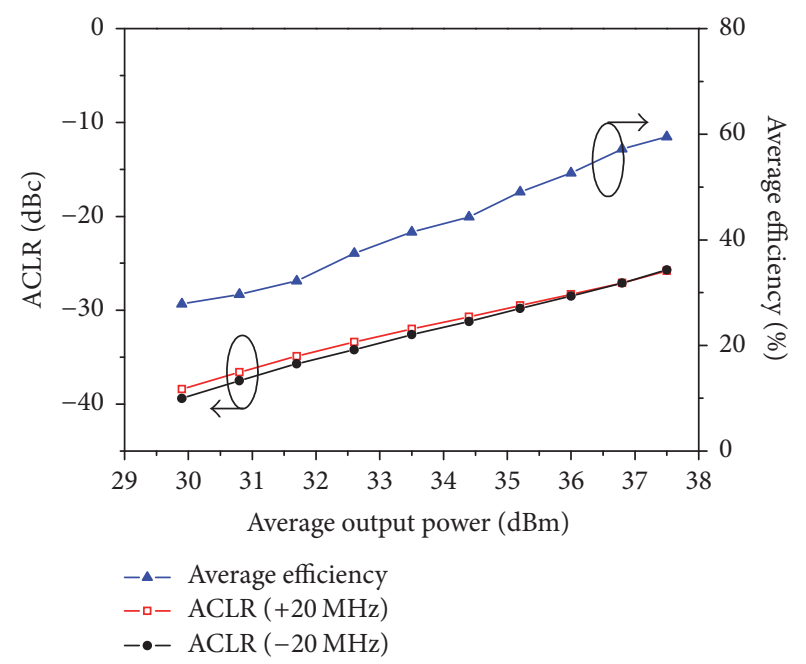

FIGURE 17: Measured average efficiencies and ACLRs for a $20 \mathrm{MHz}$ LTE signal at the frequency of $2.35 \mathrm{GHz}$.

appropriate consistency over large BOP range, especially at the frequency band of $2.2-2.4 \mathrm{GHz}$.

To evaluate the performance of the proposed DPA for modulated signals application, a $20 \mathrm{MHz}$ LTE signal with PAPR of about $8 \mathrm{~dB}$ was used to assess the efficiency and linearity performances at $2.35 \mathrm{GHz}$. Figure 17 depicts the measured average efficiencies and adjacent channel leakage ratios (ACLRs) versus the average output powers. The DPA achieved $50 \%$ average efficiency at $9 \mathrm{~dB}$ BOP, while ACLR is around $-30 \mathrm{dBc}$. Then, the DPA was linearized using the digital predistortion (DPD) technique used in [21]. The measured power spectral density (PSD) before and after $\mathrm{DPD}$ at $9 \mathrm{~dB}$ BOP is shown in Figure 18. Well-linearized ACLR of about $-53 \mathrm{dBc}$ can be obtained. The result shows that the distortion caused by the DPA's nonlinearities and memory effects can be effectively removed. To validate the linearity improvement over the whole frequency band, the DPD linearization was implemented at the frequencies of 2.2 , 
TABLE 1: Comparison with reported large back-off DPAs.

\begin{tabular}{|c|c|c|c|c|c|c|}
\hline Ref. & Type & $\begin{array}{l}\text { Freq. } \\
(\mathrm{GHz})\end{array}$ & $\begin{array}{c}\text { Effi.@ } \\
\text { Sat. (\%) }\end{array}$ & $\begin{array}{c}\text { Effi.@ } \\
9 \text { dB BOP (\%) } \\
\end{array}$ & $\begin{array}{c}\text { Signal BW } \\
(\mathrm{MHz})\end{array}$ & $\begin{array}{r}\text { ACLR } \\
(\mathrm{dBc}) \\
\end{array}$ \\
\hline $2015[22]^{1}$ & Asym. & $2.0-2.7$ & $58-70$ & $45-66$ & 20 & $-39^{4}$ \\
\hline $2014[17]^{2}$ & Sym. & $1.9-2.3$ & $65-70$ & $40-55$ & 20 & -49 \\
\hline $2016[18]^{1}$ & Sym. & $1.95-2.05$ & $70-75$ & 53 & 5 & $-23^{3}$ \\
\hline $2017[19]^{1}$ & Sym. & $2.2-2.3$ & $62.9-71$ & $45.1-49.6$ & 20 & -51 \\
\hline T. W. ${ }^{1}$ & Sym. & $2.2-2.5$ & $63-68$ & $45-52$ & 20 & -53 \\
\hline
\end{tabular}

Asym.: asymmetric DPA, Sym.: symmetric DPA. ${ }^{1}$ Packaged device. ${ }^{2}$ Bare-die device. ${ }^{3}$ Without DPD. ${ }^{4}$ With memoryless DPD.

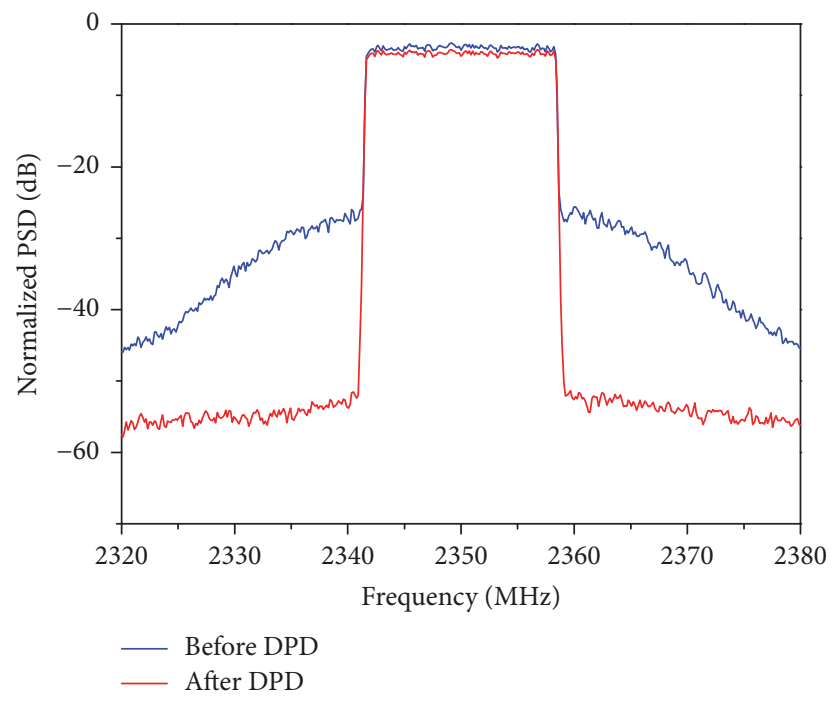

FIGURE 18: Measured power spectral density with the LTE signal at $9 \mathrm{~dB}$ BOP before and after DPD.

2.3, 2.4, and $2.5 \mathrm{GHz}$. Figure 19 gives the measured output powers, average efficiencies, and ACLRs versus frequencies at about $9 \mathrm{~dB}$ BOP. It is shown that the DPA can achieve good average efficiency of around $50 \%$ with ACLR of about $-50 \mathrm{dBc}$ after linearization.

For validation of the designed DPA for dual-band operations, a dual-band modulated signal with bandwidths of 10 and $20 \mathrm{MHz}$ at 2.25 and $2.45 \mathrm{GHz}$, respectively, was employed to assess the efficiency and linearity performances of the DPA. The two dimensional decomposed vector rotation-based model was used for linearization [23, 24]. The measured PSD before and after DPD at the average output power of $33.7 \mathrm{dBm}$ with associated efficiency of $43 \%$ is shown in Figure 20. It can be observed that the ACLRs are lower than -45 and $-51 \mathrm{dBc}$ for the first and second band, respectively. The linearization results show that good linearity and efficiency performance of the designed DPA can be achieved for multistandard wireless communication systems. Table 1 shows the performance comparison with published DPAs. When compared with most of the reported DPAs with good linearity, the proposed symmetric DPA using IER can provide high efficiency at $9 \mathrm{~dB}$ BOP and achieve wideband operation simultaneously.

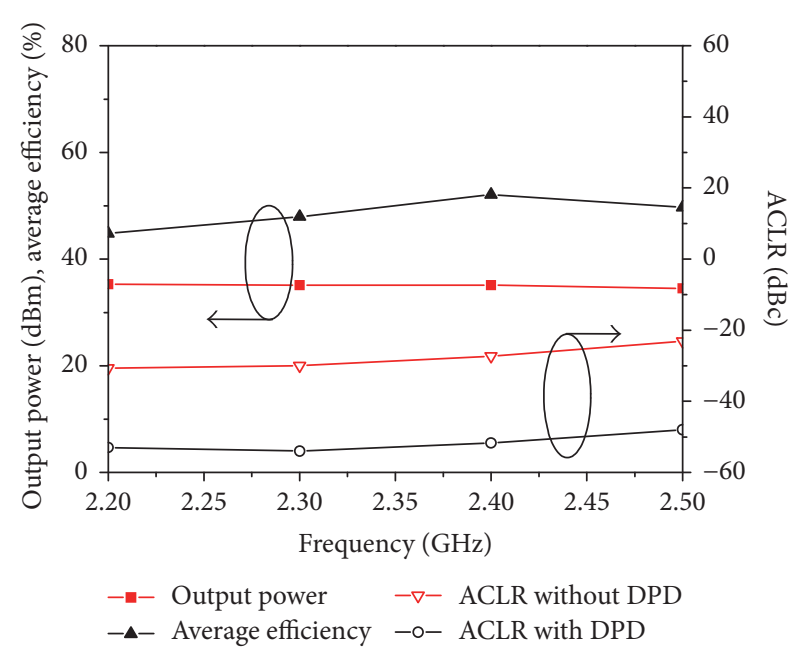

FIGURE 19: Measured output powers, average efficiencies, and ACLRs versus frequencies at about $9 \mathrm{~dB}$ BOP.

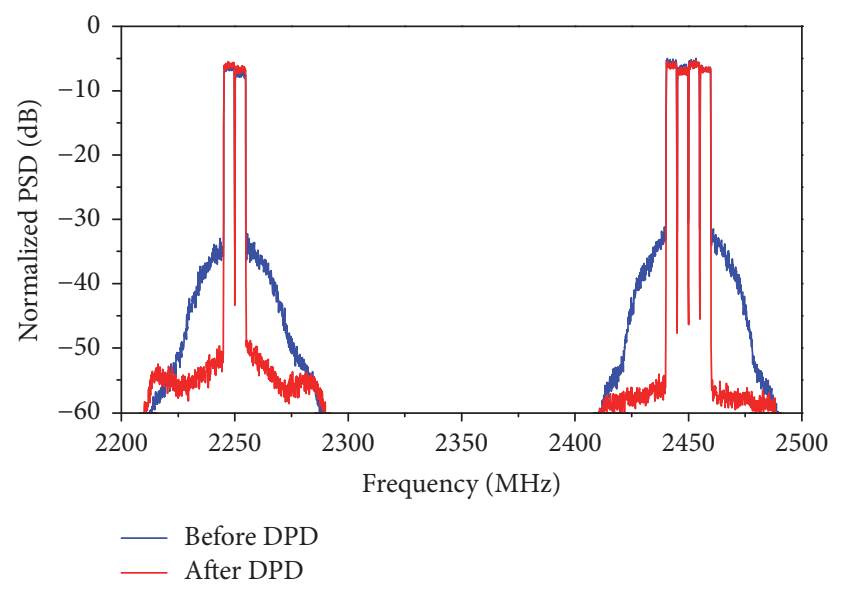

FIGURE 20: Measured power spectral density with the dual-band modulated signal before and after DPD.

\section{Conclusion}

A DPA design approach for BOP range extension using IER was proposed. A 2.2-2.5 GHz symmetric DPA with $9 \mathrm{~dB}$ BOP range was developed and measured. Experimental results show that the proposed DPA has the drain efficiency of higher 
than $45 \%$ at $9 \mathrm{~dB}$ BOP over a wide frequency band, which can satisfy the efficiency requirement when the DPA is driven by modern modulated signals with PAPRs of higher than $6 \mathrm{~dB}$.

\section{Conflicts of Interest}

The authors declare that they have no conflicts of interest.

\section{Acknowledgments}

This work was supported by the National Science Foundation of China under Grant no. 61701199 and the Natural Science Foundation of Jiangsu Province of China under Grant no. BK20150528. This work was also supported by the Foundation of the State Key Laboratory of Millimeter Waves under Grant no. K201816 and the Natural Science Foundation of the Jiangsu Higher Education Institutions of China under Grants nos. 15KJB510007 and 16KJB510006. This work was also supported by the Research Foundation for Advanced Talents of Jiangsu University under Grant no. 15JDG080.

\section{References}

[1] A. Grebennikov and S. Bulja, "High-efficiency doherty power amplifiers: Historical aspect and modern trends," Proceedings of the IEEE, vol. 100, no. 12, pp. 3190-3219, 2012.

[2] K. Rawat and F. M. Ghannouchi, "Design methodology for dual-band doherty power amplifier with performance enhancement using dual-band offset lines," IEEE Transactions on Industrial Electronics, vol. 59, no. 12, pp. 4831-4842, 2012.

[3] S. Chen and Q. Xue, "Compact triple-transistor doherty amplifier designs: Differential/power combining," IEEE Transactions on Microwave Theory and Techniques, vol. 61, no. 5, pp. 19571963, 2013.

[4] A. M. M. Mohamed, S. Boumaiza, and R. R. Mansour, "Electronically tunable doherty power amplifier for multi-mode multi-band base stations," IEEE Transactions on Circuits and Systems I: Regular Papers, vol. 61, no. 4, pp. 1229-1240, 2014.

[5] J. Lee, J. Son, and B. Kim, "Optimised Doherty power amplifier with auxiliary peaking cell," IEEE Electronics Letters, vol. 50, no. 18, pp. 1299-1301, 2014.

[6] R. Kalyan, K. Rawat, and S. K. Koul, "Design strategy of concurrent multi-band Doherty power amplifier," IET Microwaves, Antennas \& Propagation, vol. 9, no. 12, pp. 1313-1322, 2015.

[7] C. Musolff, M. Kamper, Z. Abou-Chahine, and G. Fischer, "A linear and efficient doherty PA at $3.5 \mathrm{GHz}$, IEEE Microwave Magazine, vol. 14, no. 1, pp. 95-101, 2013.

[8] L. Piazzon, R. Giofre, P. Colantonio, and F. Giannini, "A method for designing broadband doherty power amplifiers," Progress in Electromagnetics Research, vol. 145, pp. 319-331, 2014.

[9] C. Musolff, M. Kamper, Z. Abou-Chahine, and G. Fischer, "Linear and efficient Doherty PA Revisited," IEEE Microwave Magazine, vol. 15, no. 1, pp. 73-79, 2014.

[10] J. Xia and X. Zhu, "Doherty power amplifier with enhanced inband load modulation for $100 \mathrm{MHz}$ LTE-advanced application," Microwave and Optical Technology Letters, vol. 57, no. 2, pp. 391395, 2015.

[11] X. Chen, W. Chen, F. M. Ghannouchi, Z. Feng, and Y. Liu, "A broadband doherty power amplifier based on continuousmode technology," IEEE Transactions on Microwave Theory and Techniques, vol. 64, no. 12, pp. 4505-4517, 2016.
[12] X. Y. Zhou, S. Y. Zheng, W. S. Chan, S. Chen, and D. Ho, "Broadband Efficiency-Enhanced Mutually Coupled Harmonic Postmatching Doherty Power Amplifier," IEEE Transactions on Circuits and Systems I: Regular Papers, vol. 64, no. 7, pp. 17581771, 2017.

[13] S. H. Chun, D. H. Jang, J. Y. Kim, and J. H. Kim, "Inverted asymmetric Doherty power amplifier driven by two-stage symmetric Doherty amplifier," IEEE Electronics Letters, vol. 46, no. 17, pp. 1208-1209, 2010.

[14] L. Piazzon, P. Colantonio, F. Giannini, and R. Giofré, "Asymmetrical Doherty power architecture with an integrated driver stage in the auxiliary branch," International Journal of RF and Microwave Computer-Aided Engineering, vol. 24, no. 4, pp. 498507, 2014.

[15] J. Xia, M. Yang, and A. Zhu, "Improved Doherty Amplifier Design with Minimum Phase Delay in Output Matching Network for Wideband Application," IEEE Microwave and Wireless Components Letters, vol. 26, no. 11, pp. 915-917, 2016.

[16] H. Kang, H. Lee, H. Oh et al., "Symmetric Three-Way Doherty Power Amplifier for High Efficiency and Linearity," IEEE Transactions on Circuits and Systems II: Express Briefs, vol. 64, no. 8, pp. 862-866, 2017.

[17] X. H. Fang and K.-K. M. Cheng, "Extension of high-efficiency range of doherty amplifier by using complex combining load," IEEE Transactions on Microwave Theory and Techniques, vol. 62, no. 9, pp. 2038-2047, 2014.

[18] M. Özen, K. Andersson, and C. Fager, "Symmetrical Doherty Power Amplifier with Extended Efficiency Range," IEEE Transactions on Microwave Theory and Techniques, vol. 64, no. 4, pp. 1273-1284, 2016.

[19] W. Shi, S. He, and N. Gideon, "Extending high-efficiency power range of symmetrical Doherty power amplifiers by taking advantage of peaking stage," IET Microwaves, Antennas \& Propagation, vol. 11, no. 9, pp. 1296-1302, 2017.

[20] W. Shi, S. He, F. You et al., "The Influence of the Output Impedances of Peaking Power Amplifier on Broadband Doherty Amplifiers," IEEE Transactions on Microwave Theory and Techniques, vol. 65, no. 8, pp. 3002-3013, 2017.

[21] J. Xia, M. Yang, Y. Guo, and A. Zhu, "A broadband highefficiency Doherty power amplifier with integrated compensating reactance," IEEE Transactions on Microwave Theory and Techniques, vol. 64, no. 7, pp. 2014-2024, 2016.

[22] X. A. Nghiem, J. Guan, and R. Negra, "Broadband Sequential Power Amplifier with Doherty-Type Active Load Modulation," IEEE Transactions on Microwave Theory and Techniques, vol. 63, no. 9, pp. 2821-2832, 2015.

[23] N. Kelly, W. Cao, and A. Zhu, "Preparing Linearity and Efficiency for 5G: Digital Predistortion for Dual-Band Doherty Power Amplifiers with Mixed-Mode Carrier Aggregation," IEEE Microwave Magazine, vol. 18, no. 1, pp. 76-84, 2017.

[24] Y. Guo and A. Zhu, "Power adaptive decomposed vector rotation based digital predistortion for RF power amplifiers in dynamic power transmission," in Proceedings of the 2017 IEEE Topical Conference on Power Amplifiers for Wireless and Radio Applications, PAWR 2017, pp. 8-10, USA, January 2017. 


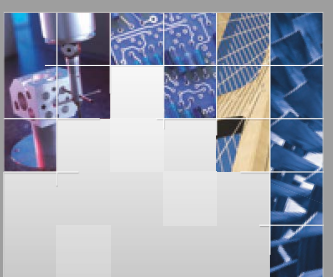

\section{Enfincering}
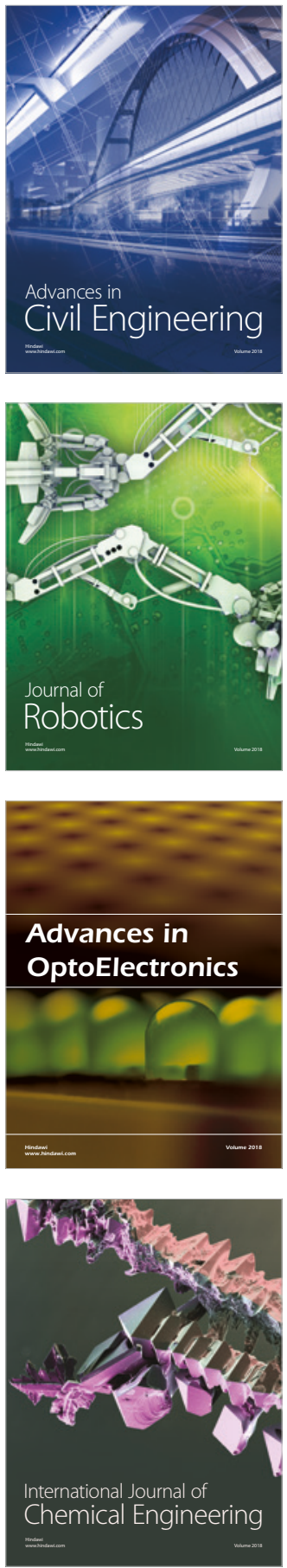

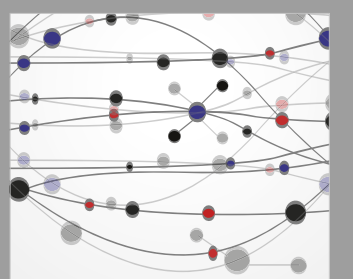

\section{Rotating \\ Machinery}

The Scientific World Journal

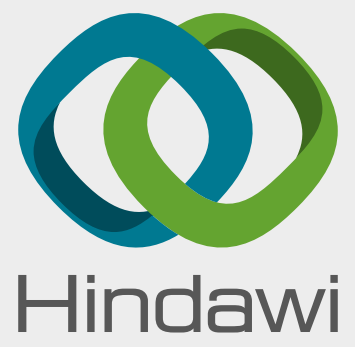

Submit your manuscripts at

www.hindawi.com
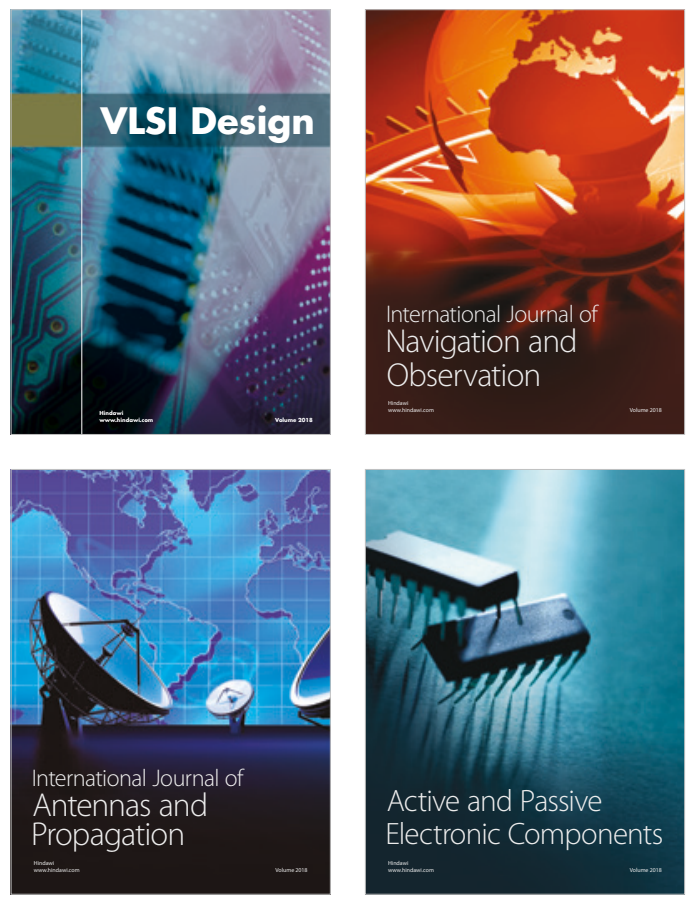
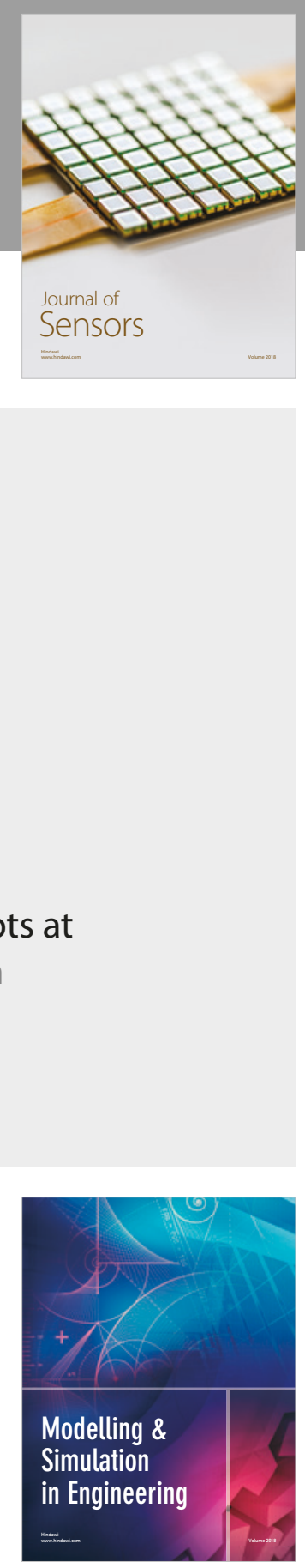

\section{Advances \\ Multimedia}
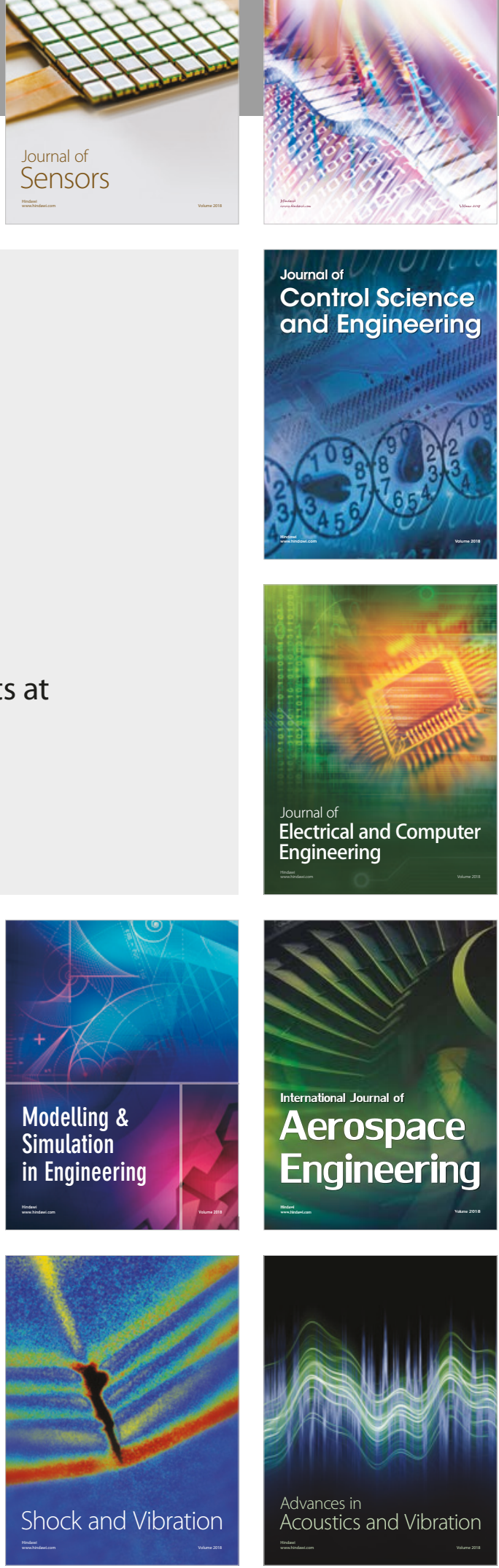